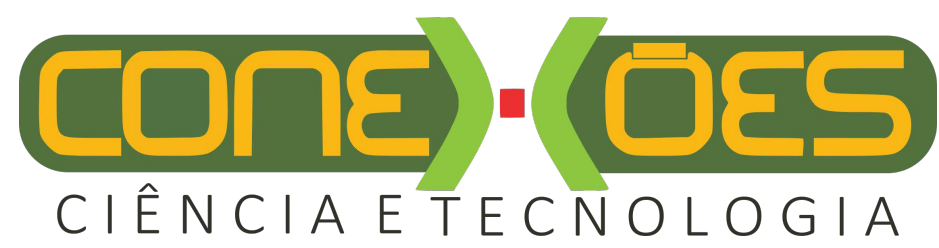

\title{
A RELATIVIDADE GERAL E A BUSCA DE NOVAS DIMENSÕES
}

\author{
LEONARDO OSPEDAL PRESTES ROSAS, JOSÉ ABDALLA HELAYËL-NETO
}

\author{
Centro Brasileiro de Pesquisas Físicas (CBPF) \\ Rio de Janeiro, Brasil \\ <leoopr@cbpf.br>,<helayel@cbpf.br> \\ DOI: $10.21439 /$ conexoes.v13i2.1661
}

\begin{abstract}
Resumo. A proposta da presente contribuição é descrever como a Relatividade Geral, na qualidade de uma teoria elaborada para investigar a estrutura do espaço-tempo e suas propriedades geométricas, estimulou, no século passado, a introdução de novas dimensões de natureza espacial e temporal para a nossa compreensão das interações fundamentais, possibilitando um harmoniosa junção da micro com a macrofísica.
\end{abstract}

Palavras-chaves: Relatividade Geral. Dimensões extras. Interações fundamentais

\begin{abstract}
This contribution sets out to describe how General Relativity, as a theory to inspect the spacetime strucuture and its geometrical properties, triggered, throughout last century, the idea of new spaceand time-like dimensions to understand features of fundamental interactions, allowing a perfect matching between micro- and macrophysics.
\end{abstract}

Keywords: General Relativity. Extra-dimensions. Fundamental interactions.

A Teoria da Relatividade Geral (TRG), proposta por Einstein em 1915 (EINSTEIN, 1915), não só incorporou a abordagem de Newton para o campo gravitacional, mas, sobretudo, apresentou previsões de longo alcance, como o desvio para o vermelho de origem gravitacional, a precessão do periélio da órbita de Mercúrio, a deflexão de feixes de luz em um campo de gravidade, a existência de ondas gravitacionais e de buracos negros. Estas duas últimas antecipações tiveram a sua descoberta anunciada em Fevereiro de 2016, portanto, antevisões que aguardaram um século a sua comprovação. De fato, a observação das ondas gravitacionais produzidas pela fusão de dois buracos negros, um de 36 e outro de 29 massas solares constitui-se, na época, como a mais concreta evidência da existência de buracos negros (ABBOT, 2016). Muito importante também destacar o impacto da Relatividade Geral para a Cosmologia no celebrado trabalho de Einstein de 1917 (EINSTEIN, 1917), quando apresenta e discute as implicações cosmológicas da TRG. Nasce, assim, a Cosmologia como uma nova área da Física. Vê-se, mediante estes fatos, estar-se diante de uma grande teoria científica, que incorpora teorias e modelos anteriores, introduz uma nova formulação matemática na Física (a Geometria Riemanniana) e faz previsões que aguardam até um século para serem comprovadas.
Neste ano de 2019, a Física comemora os cem anos do célebre experimento realizado em Sobral e que se constituiu no primeiro grande teste experimental da TRG. Com este propósito, participamos desta comemoração contribuindo para a publicação deste volume com uma discussão que foi plenamente ampliada pela Relatividade Geral e que repercute fortemente até os nossos dias: a introdução de dimensões espaciais e temporais suplementares às quatro dimensões espaço-temporais que percebemos. A possibilidade de unificação das interações eletromagnética e gravitacional, as duas únicas estabelecidas antes dos anos 1920, foi o ponto de partida para a adoção de dimensões extras na Física. E este é o caminho que escolhemos percorrer com a nossa contribuição a esta publicação.

É importante deixar claro que o nosso objetivo é traçar uma trajetória descritiva e cronológica da introdução de dimensões suplementares na formulação de teorias e modelos para as interações fundamentais. Ressaltamos que não será uma revisão dos aspectos técnicos. Optamos por mostrar como os fatos relacionados às dimensões extras foram sendo incorporados com o avanço da Teoria Quântica de Campos, a partir dos anos 1950, em conexão com a Física de Partículas e com aspectos ligados ao programa de unificação das interações fundamentais. 


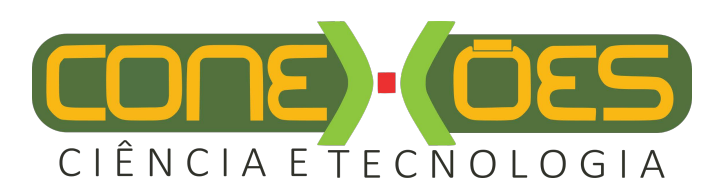

A RELATIVIDADE GERAL E A BUSCA DE NOVAS DIMENSÕES

Como método de trabalho, estaremos colocando em destaque as propostas que foram sendo feitas e citando os trabalhos seminais onde as ideias foram lançadas.

A Física de Interações Fundamentais vem experimentando um sempre reavivado interesse pelo estudo de teorias de campos definidas em espaços-tempo com dimensões mais altas do que quatro. O nosso ponto de vista é que estes modelos com mais dimensões sejam encarados como teorias efetivas no regime de baixas energias de alguma teoria genuinamente mais fundamental como, por exemplo, as Supercordas, e se propõem a investigar diferentes mecanismos que possam resolver problemas nada triviais do Modelo-Padrão das Partículas Elementares. Entretanto, já há quase um século, a proposta de teorias físicas em dimensionalidades acima de quatro já motivava a área, sobretudo com o propósito maior de buscar um possível cenário unificado para as interações gravitacional e eletromagnética. Deve-se chamar atenção para o fato de que uma questão comum a todos os modelos desenvolvidos em dimensões mais altas é a necessidade de se justificar por que as dimensões extra se desacoplam das quatro dimensões espaço-temporais que experimentamos.

O ponto de vista mais tradicionalmente adotado é se supor que estas dimensões suplementares sejam compactas, com uma escalas de comprimento tão pequenas que não sejam acessíveis nos níveis de energia dos presentes aceleradores, não podendo, portanto, ser diretamente detectáveis. (Entretanto, a partir de 1999 com os trabalhos seminais de Randall e Sundrum - este ponto de vista, mais universalmente seguido, pode ser evitado, considerando-se as chamadas "warped geometries"com gravitação localizada. Nesta proposta as dimensões extras não precisam ser compactas e estarem em escala Planckeana, podendo ser de dimensões acessíveis ou até mesmo não-compactas. Faremos referência posteriormente a esta nova abordagem ao problema das dimensões extra.)

Iniciemos, contudo, a nossa apresentação, retornando às origens da proposta de mais dimensões, em 1914, Nordström (NORDSTRÖM, 1914), tendo como objetivo elaborar um cenário unificado para o eletromagnetismo e um campo gravitacional escalar, introduz a ideia de uma quinta dimensão. Este trabalho deve ser considerado como o marco inicial da proposta de dimensões extras em Física. Nesta linha, Kaluza adotou, em 1921, a ideia de Nordström de uma dimensão espacial suplementar (KALUZA, 1921), tendo também em vista a proposta de unificar a gravitação (agora, na formulação Einsteiniana, portanto, com um campo gravitacional tensorial, não mais escalar) e o eletromagne- tismo. Unificação, neste contexto, significa que, apesar de todas as suas diferenças, estas duas interações podem ser compreendidas como tendo uma origem em comum. Desde então, a proposta de se entender geometricamente os números quânticos internos (como a carga elétrica, por exemplo) ganhou adesão na comunidade das interações fundamentais. Da mesma forma que energia e momento são descritos em conexão com as translações espaço-temporais, o que se propunha era compreender os números quânticos internos e as interações a estes associadas através de simetrias ligadas a dimensões extra.

Em 1926, independentemente, três autores - Klein (KLEIN, 1926), Mandel (MANDEL, 1926) e Fock (FOCK, 1926) - retomam a iniciativa de Nordström e Kaluza, propondo que a interação eletromagnética deixasse de ter o status de uma interação especial e passasse a ser vista simplesmente como uma componente da interação gravitacional, com o sistema gravitacional, porém, em uma dimensão extra. Em 1938, Klein (KLEIN, 1938) apresenta, na épica Conferência New Theories in Physics, realizada em Varsóvia, o seu notável seminário On the theory of charged fields ${ }^{\sim}$ que seria a primeira versão do que chamamos atualmente The Theory of Everything $`$ no qual notavelmente unificava todas as partículas conhecidas desde o elétron até então (incluindo aí os hipotéticos mésons $-\pi$ previstos por Yukawa em 1935) em uma descrição unificada das interações gravitacional, eletromagnética e nucleares (forte e fraca) em um espaço-tempo 5-dimensional (YUKAWA, 1935).

No mesmo ano de 1938, Bergmann e Einstein (BARGMANN; EINSTEIN, 1938) introduzem um elemento novo a partir da proposta de Kaluza e Klein: compreender a quantização da carga elétrica de uma forma diferente do que Paul Dirac havia proposto em seu trabalho de 1931 (DIRAC, 1931). Bergmann e Einstein associam a simetria $U(1)$ do eletromagnetismo à ideia de uma quinta dimensão compacta e esta propriedade e a compaticidade da quinta dimensão justifica a simetria eletromagnética $U(1)$ bem como a natureza quantizada da carga elétrica. A geometrização da carga em associação com uma quinta dimensão compacta garante a quantização da carga elétrica.

Após a considerável interrupção, devido à II Grande Guerra, no fluxo das ideias que avançavam no riquíssimo período 1925-1938, a área das interações fundamentais em associação com a teoria quântica de campos é retomada no pós-guerra, mas com direcionamento para questões das propriedades físicas e as interações entre as chamadas partículas elementares. Os mésons $-\pi$ de Yukawa são descobertos em 1947, a com- 


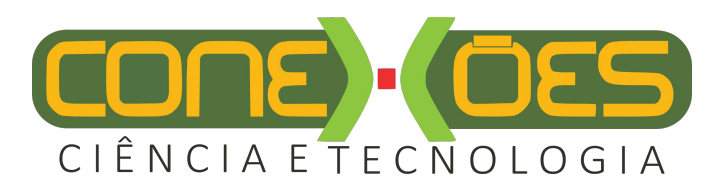

A RELATIVIDADE GERAL E A BUSCA DE NOVAS DIMENSÕES

preensão da Eletrodinâmica Quântica avança com interesse renovado e a Física Hadrônica, com o desenvolvimento dos aceleradores de partículas, passa a ser dominante na área das Interações Fundamentais. Neste cenário, as ideias de Kaluza-Klein deixam de ter o protagonismo que haviam conquistado na década de 1930.

Apesar do hiato que se preanunciava para as chamadas Teorias de Kaluza-Klein, é importante destacar que a ideia de se geometrizar as interações (agora, na década de 1950, é a vez das interações nucleares) não está completamente esquecida. Shaw e Salam (SHAW, 1954) e, contemporaneamente, Yang-Mills concebem, em 1954, as chamadas Teorias de Yang-Mills-Shaw , que se propõem a descrever, com uma abordagem mais geometrizada, a interação nuclear forte entre prótons e nêutrons, não mais mediada pela troca de bósons escalares.(os mésons $-\pi$ de Yukawa), mas, agora, em maior proximidade ao paradigma eletromagnético: a troca de bósons vetoriais (YANG; MILLS, 1954). Na formula-

ção de Yang-Mills-Shaw os próprios mésons $-\pi$ interagem nuclearmente trocando também esta nova categoria de mediadores. Em 1956, Utyiama (UTYIAMA, 1956) publica um importante trabalho, no qual sublinha de forma mais contundente a similaridade da formulação de Yang-Mills-Shaw com a descrição geométrica da gravidade em que se baseia a Teoria da Relatividade Geral. Kibble (1961), aprofunda e esclarece melhor o trabalho de Utyiama e reescreve a gravitação como uma teoria de Yang-Mills-Shaw elegendo a simetria de Lorentz da Relatividade Especial como grupo de gauge da formulação. Podemos estabelecer este marco como o final daquela que se poderia chamar fase-I das Teorias de Kaluza-Klein. Mesmo sem introduzir as dimensões extra, as Teorias de Yang-Mills-Shaw seguem a proposta de Kaluza-Klein de se geometrizar interações associadas a números quânticos internos, como carga elétrica e spin isotópico. A área entra em silêncio de novo, mas, desta vez, somente até 1976. É oportuno, a este ponto, ressaltar que, dentro do cenário de Kaluza-Klein, uma teoria de Yang-Mills pode emergir da formu-lação de um modelo de gravidade em mais altas dimensões através do chamado mecanismo de compactificação espontânea. Um trabalho claro e bastante pedagógico para a elucidação deste ponto é o artigo (SALAM, STRATHDEE, 1982), cujos apêndices são muito didáticos e mostram de forma muito clara como campos de Yang-Mills podem emergir de estruturas geométricas de espaços-tempo curvos.

O período 1962-1976 é efervescente em outras direções: muitas novas ideias, outros paradigmas, avanços na compreensão da implementação de simetrias, grandes previsões teóricas, descobertas que consagram estas previsões, completa-se a Teoria Eletrofraca de SalamGlashow-Weinberg em 1967; entra em cena a liberdade assintótica com os experimentos do SLAC em 19681969 e Gross-Wilczek e Politzer, independentemente, demonstram, em 1973, no âmbito de uma Teoria de Yang-Mills-Shaw com grupo de gauge $S U(3)$, a propriedade de liberdade assintótica dos quarks. Neste contexto, as interações eletrofracas e nucleares fortes atingem o status teórico de teorias quânticas de campos consistentes e aptas a descrever a fenomenologia do mundo das partículas genuinamente elementares (quarks, léptons carregados, neutrinos e bósons vetoriais intermediários). Temos, então, formulado o Modelo Padrão da Física de Partículas Elementares.

Deve-se, aqui, chamar atenção para o relevante trabalho de Dirac (1963), onde o autor estuda o grupo de anti-de Sitter, $S O(2,3)$, e obtém a peculiar representação dos singletons, que são restritas ao espaço de antide Sitter e não aparecem como representações do grupo de Poincaré, mesmo no limite de constante cosmológica nula. Ainda que sendo realizado em $(1+3)$ dimensões, i.e., sem a incorporação de uma dimensão extra, este trabalho merece ser citado por antecipar a ideia da correspondência $A d S / C F T$, conjecturada por Maldacena (1998) e reafirmada por Witten (1998), ao discutirem o espaço de anti-de Sitter 5-dimensional, $A d S_{5}$.

No novo cenário que se descortina, dois grande projetos ocupam a comunidade da área das interações fundamentais: a formulação consistente de uma teoria quântica da gravitação e a busca de um formalismo unificado que demonstre que todas as quatro interações (eletromagnética, nuclear fraca, nuclear forte e gravitação) têm uma origem em comum. A gravitação, de um ponto de vista mais microscópico, catalisa um grande número de esforços e isto contribui para o que ocorrerá alguns anos à frente, mais exatamente, em 1978, o renascimento das Teorias de Kaluza-Klein. A ponte que se estabelece para este ressurgimento é a introdução da Supersimetria (1973) e da Supergravidade (1976) na área das teorias para as interações fundamentais.

É justamente o aparecimento das teorias de supergravidade que restaura o interesse e estimula a busca de novos caminhos para as Teorias de Kaluza-Klein e os métodos de redução dimensional. Em 1975, Scherk e Schwarz (SCHERK; SCHWARZ, 1975) retomam os cenários multidimensionais e propõem um método de redução dimensional apropriado para estudar o espectro de campos em modelos reduzidos dimensionalmente a partir de modelos definidos inicialmente em dimensões mais altas. A redução dimensional é trivial: consiste em se considerar apenas as configurações de campos que não dependem das coordenadas espaço-temporais 


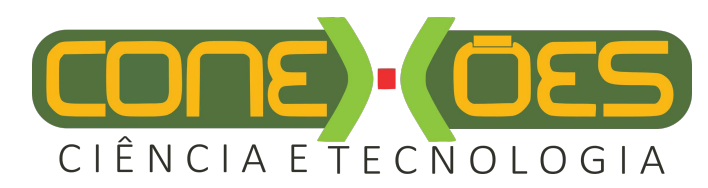

A RELATIVIDADE GERAL E A BUSCA DE NOVAS DIMENSÕES

extras; considera-se que os campos dependam apenas das coordenadas espaço-temporais do espaço de Minkowski em $4 D$. Esta prescrição funciona para o estudo do chamado setor de modos-zero (campos de massa nula) e é útil também para se chegar às simetrias internas do modelo reduzido. Entretanto, a ideia é imposta por decreto, e não segue naturalmente da dinâmica do sistema de campos

Buscando uma prescrição com mais fundamentação dinâmica, Cremmer e Scherk desenvolvem, em 1976, um novo mecanismo de redução dimensional, um passo adiante da proposta de Kaluza-Klein: a chamada redução dimensional por compactificação espontânea, na qual a dinâmica que se dá em dimensões mais altas indica, através das soluções das equações de campo, que a geometria pode ser fatorada em um espaço-tempo de (1+3) dimensões e um espaço interno $N$-dimensional, cujas isometrias se associam às simetrias de gauge do mundo quadridimensional (CREMMER; SCHERK, 1976). A compactificação espontânea abre uma nova era de efervescentes atividades na busca pela unificação e encontra uma forte base teórica na supersimetria; esta provê, através de seus supermultipletes, os campos de matéria necessários para a indução da compactificação espontânea. Sem supersimetria, a introdução destes campos torna-se um processo ad-hoc. Em um cenário de supersimetria estendida, a matéria é naturalmente introduzida nos supermultipletes de gauge ou nos supermultipletes de gravitação.

Em seguida à contribuição de Cremmer-Scherk, o período 1976-1978 foi marcado pela publicação de vários trabalhos onde se apresentavam diferentes modelos à la Kaluza-Klein baseados no fenômeno da compactificação espontânea, sempre com vistas ao projeto de unificação de todos os campos de força. Em 1978, Nahm mostra que 11 é o número máximo de dimensões permitidas pela supersimetria (NAHM, 1978) e, em 1979, Scherk e Schwarz estudam a geração de massa e um mecanismo de quebra espontânea da supersimetria por redução dimensional na formulação de compactificação espontânea da supergravidade (SCHERK; SCHWARZ, 1979), abordagem que se difundiu sob a terminologia de Supergravidades de Kaluza-Klein. Estas foram de central importância e dominaram a literatura referente à unificação e à investigação da gravitação quântica até 1987.

Em 1980, destaca-se o trabalho de Freund-Rubin (FREUND; RUBIN, 1980), que aplica o mecanismo da compactificação espontânea à supergravidade maximalmente estendida (11-dimensional): chega-se a um espaço-tempo quadridimensional com constante cosmológica negativa, isto é, um espaço-tempo de anti-de
Sitter $(A d S)$. Este trabalho é a base para a compreensão da relação entre supersimetria e espaços da categoria $A d S$. Cabe ressaltar também que, em 1980, Orzalesi publica um importante trabalho de revisão crítica sobre a unificação do ponto de vista das Teorias de KaluzaKlein (ORZALESI, 1980).

Em 1981, Witten publica um trabalho indicando que 11 dimensões, além de ser o espaço-tempo mais amplo possível para acomodar a supersimetria, é também o número mínimo de dimensões para acomodar um grupo de isometria $S U(3) \times S U(2) \times U(1)$, que nada mais é do que o grupo de gauge do Modelo Padrão (WITTEN, 1981). A este ponto, ainda que rompendo com a ordem cronológica dos fatos, cabe destacar que, a par-tir desta proposta de física em 11 dimensões, Witten propõe, em 1995, uma formulação mais completa, a chamada Teoria-M (WITTEN,1995), a partir da qual as diferentes teorias de supercordas (sobre es-tas, falaremos mais à frente) podem ser obtidas. A introdução da Teoria-M marca o período que se cha-mou a Segunda Revolução das Cordas. Logo em se-guida, como um desdobramento, Vafa introduz, em 1996, a Teoria-F, esta formulada em 12 dimensões (VAFA, 1996; WEIGAND, 2018).

Recebeu notável atenção o trabalho de Percacci e Randjbar-Daemi, onde os autores mostram que o mecanismo de compactificação espontânea, associado à presença de campos de Yang-Mills já introduzidos em altas dimensões, pode eliminar a constante cosmológica do mundo em $(1+3)$ dimensões e ter como solução o produto cartesiano entre um espaço-tempo plano quadridimensional (Minkowsiki), no lugar de um espaço $A d S$, e um espaço compacto $\mathrm{N}$-dimensional, como pressu-põe o mecanismo (PERCACCI; RANDJBAR-DAEMI, 1982).

Em 1980, uma novidade no que diz respeito às Teorias de Kaluza-Klein: um método de redução dimensional alternativo e radicalmente diferente dos esquemas de Scherk-Schwarz e do fenômeno de compactificação espontânea: a chamada redução dimensional por transformação de Legendre, desenvolvido por Sohnius, Stelle e West (SOHNIUS; STELLER; WEST, 1980). Este processo não omite dependência nas coordenadas espaciais extras, também não as compactifica e não se baseia em uma solução clássica para os campos que definem o modelo em dimensões mais altas. $\mathrm{O}$ esquema de Sohnius-Stelle-West é uma proposta realmente nova e se mostra muito conveniente para a obtenção de modelos supersimétricos estendidos com supersimetria realizada off-shell, que é uma questão não trivial (veja, por exemplo, PATRICK, 2006 ).

Um significativo avanço no estuda das dimensões 


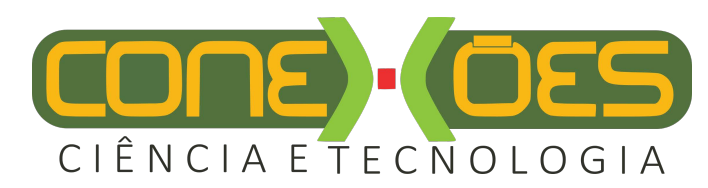

A RELATIVIDADE GERAL E A BUSCA DE NOVAS DIMENSÕES

extras é feito em Maio de 1984, em um notável trabalho de Sakharov: as dimensões suplementares são, agora, do tipo-tempo, novos eixos temporais. O trabalho Cosmological transitions with a change in the metric signature (SAKHAROV, 1984) levanta a hipótese de que novas coordenadas temporais podem ser geradas por meio de transições quânticas que induzem uma alteração na assinatura (no trabalho de Sakharov, definida como o número de dimensões temporais) da métrica espaço-tempo. A teoria de gravidade que o autor propõe com esta nova propriedade é uma teoria da tipo Kaluza-Klein com a propriedade especial de ser conformalmente invariante. Logo após o lançamento desta hipótese, Aref'eva e Volovich, a reforçam publicando um consequente trabalho na linha das teorias de KaluzaKlein em dimensões mais altas onde, agora, são estudadas as consequências de natureza física e os aspectos matemáticos dos espaços-tempo onde há dimensões temporais compactificadas (AREF'EVA; VOLOVICH, 1985).

É instrutivo destacar, a este ponto, que a discussão de modelos físicos com duas ou mais dimensões tipo-tempo ressurge, em bases muito diferentes daquelas estabelecidas por Sakharov e seus colaboradores, alguns anos depois, já na década de 1990, em conexão com a Supersimetria e a Supergravidade formuladas nos chamados espaços de Atiyah-Ward (linha fortemente trabalhada por Jim Gates e colaboradores (KETOV; NISHINO, GATES, 1993) e também problema da compactificação das Teorias de Supercordas (este último desenvolvimento já em final dos anos 1990 e início dos anos 2000). Para se ter uma ideia sistematizada e global de modelos físicos com tempos suplementares, deixamos como referência o trabalho de Foster e Müller (FOSTER, MÜLLER, 2010), Physics with two time dimensions. A literatura citada por este trabalho cobre os desenvolvimentos mais importantes feitos na linha dos dimensões temporais extras.

Uma outra contribuição que deve ser destacada é o trabalho de Dolan e Duff, em 1984, onde os autores mostram, pela primeira vez, a conexão entre a receita de Kaluza-Klein e o aparecimento de álgebras de dimensão infinita, do tipo Kac-Moody, ao analisarem as simetrias dos modelos reduzidos em 4 dimensões incluindo os estados massivos juntamente aos tradicionais modos-zero (DOLAN; DUFF, 1984). Foi um outro importante passo nos anos das Supergravidades e Cosmologias de Kaluza-Klein. Com respeito a este autor, cabe a este ponto citar o seu excelente texto sobre o mundo 11-dimensional, no qual, entre vá-rios aspectos que aborda, discute a conexão entre modelos de membranas em 11 dimensões e Mecâni- -ca Quântica Supersimétrica, o que possibilita ter informações sobre a Teoria-M a partir de modelos mecânicos, isto é, a partir de uma formulação para 0-branas (DUFF,2001).

Os quase dez anos de avanços nesta frente de trabalhos, que constituem a fase-II das Teorias de KaluzaKlein, estão sumarizados e descritos, de forma muito didática, em um artigo de revisão assinado por Duff, Nilsson e Pope (1986) e em um livro publicado por Appelquist, Chodos e Freund (1987). Segue-se, a partir da fase-II, a era das compactificações, dos espaços de Calabi-Yau, dos orbifolds e da busca por configurações de vácuo das teorias de supercordas, o que se inaugura com o célebre trabalho Vacuum Configurations for Superstrings, de Candelas, Horowitz, Strominger e Witten (CANDELAS et al., 1985), no qual são discutidas também várias questões de interesse para uma fenomenologia possível além do Modelo-Padrão, baseada em supersimetria. Os anos de 1984 e 1985 assinalam a chamada Primeira Revolução das Cordas, quando, em um trabalho altamente consequente, Green e Schwarz (GREEN,SCHWARZ,1984,1985) mostram que uma teoria de Yang-Mills supersimétrica acoplada a um modelo de supergravidade simples em 10 dimensões é livre de anomalias de gauge e anoma-lias gravitacionais para os grupos de gauge especiais $\mathrm{SO}(32)$ e E8 x E8. Este trabalho abriu, no Outono de 1984, a era das supercordas. A partir daí, como já anteriormente mencionado, dimensões mais altas, especificamente, 11 e 12 foram adotadas para a for-mulação das teorias-M e $-\mathrm{F}$, respectivamente.

A literatura das Teorias de Kaluza-Klein é mais marcadamente dominada pelo estudo de aspectos geométricos e clássicos. O passo essencial é escrever um modelo clássico com as simetrias desejadas e que realize algum esquema de unificação que se esteja procurando. Mas, cumprida esta primeira etapa, é de fundamental relevância se compreender como os resultados clássicos - a estabilidade do estado de vácuo, o espectro de massas e as relações entre as constantes de acoplamento - reagem frente à inclusão dos efeitos quânticos. E, a este ponto abrem-se duas frentes de ataque ao problema: decidir se a quantização será realizada nas dimensões superiores, visando a posterior conexão com a arena quadridimensional, ou se será investigada após algum esquema de redução dimensional ter ocorrido, considerando-se, então, todos os campos e interações do modelo nas quatro dimensões. A escolha de um ou de outro procedimento pode ser irrelevante do ponto de vista puramente clássico, mas, com o processo de quantização, o procedimento pode não ser comutativo: quantizar-e-reduzir pode levar a resultados distintos de reduzir-e-quantizar. 


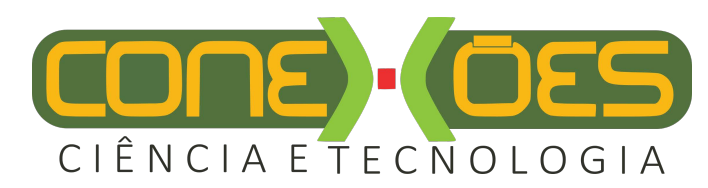

A RELATIVIDADE GERAL E A BUSCA DE NOVAS DIMENSÕES

Na direção de se considerar efeitos quânticos em Teorias de Kaluza-Klein, chamamos atenção para os artigos de Appelquist e Chodos (1983), onde os autores computam o potencial efetivo a 1-loop em termos da componente extra da métrica em 5 dimensões; os autores consideram o modelo 5-dimensional de KaluzaKlein para unificar gravitação e eletromagnetismo e obtêm o resultado a 1-loop em cinco dimensões. A partir deste resultado, mostram que a dimensão extra tende a se contrair a um tamanho na escala do comprimento de Planck. Estes dois trabalhos, na verdade, descrevem a contrapartida gravitacional do Efeito Casimir da Eletrodinâmica. Podemos dizer que Appelquist e Chodos iniciam a fase da quantização das Teorias de KaluzaKlein. Ainda em 1983, Appelquist, Chodos e Myers mostram como efeitos quânticos podem gerar instabilidades no processo de redução dimensional (ALPPEQUIST; CHODOS; MYERS, 1983).

Em seguida, Chodos (1984a) apresenta um trabalho mais genérico, onde tece várias considerações sobre as Teorias de Kaluza-Klein e, em particular, faz algumas digressões sobre os aspectos quânticos destas teorias (CHODOS, 1984b). Ainda em 1984, Candelas e Weinberg propõem a chamada redução dimensional auto-consistente e partem de $(4+\mathrm{N})$ dimensões, onde consideram um sistema de gravitação acoplada a campos de matéria que não auto-interagem e cujo tensor energia-momento corrigido à ordem de 1-loop é o responsável pela curvatura da variedade compacta $\mathrm{N}$ dimensional que emerge das $\mathrm{N}$ dimensões extras (CANDELAS; WEINBERG, 1984). Neste esquema, os autores também apresentam um procedimento para se calcular cargas e constantes de acoplamento de gauge do modelo reduzido 4-dimensional. Posteriormente, Delbourgo e Weber (1986) também estudam efeitos das correções quânticas diretamente em 5 dimensões (DELBOURGO; WEBER, 1986). Derivam os propagadores do gráviton e do fóton e o efeito dos modos massivos de spin-2 no espaço-tempo 5-dimensional, baseandose fortemente na proposta de Appelquist e Chodos para tratar a questão.

Cabe destacar também o trabalho de Huggins e Toms (1985), onde computam, no modelo 5dimensional, uma ação efetiva a 1-loop para discutir a componente Maxwelliana desta ação efetiva induzida como efeito das correções de 1-loop. De um ponto de vista sincrônico, foi uma contribuição de significado para a época.

Retomando de forma mais enfática o que colocamos acima, a tarefa de executar a quantização e computar correções quânticas no âmbito das Teorias de KaluzaKlein coloca-nos frente à duas possibilidades: realiza- se o programa no espaço-tempo completo com dimensão $D>4 \mathrm{e}$, em seguida, reduz-se o modelo com os efeitos quânticos já computados nas dimensões mais altas; ou, alternativamente, procede-se inicialmente à redução e, uma vez que o estado de vácuo, os modos-zero, as flutuações massivos e todos os acoplamentos tenham sido determinados em $D=4$, passa-se à quantização e ao cômputo dos efeitos quânticos já no espaço-tempo reduzido quadridimensional. Trabalhos publicados sobre a questão, e que serão citados mais à frente, mostram que pode, de fato, haver uma espécie de inequivalência quântica entre modelos de Kaluza-Klein quando se adota um ou outro ponto de vista. Estabelece-se, a partir dos trabalhos de Appelquist e Chodos (1983), uma rica literatura no tópico das altas dimensões, mecanismos de compactificação e efeitos quânticos das altas dimensionalidades (APPELQUIST; CHODOS, 1983a, 1983b). Optamos aqui por fazer uma varredura do tópico, de 1983 à atualidade, apenas demarcando o aparecimento daquelas ideias - e as correspondentes referências seminais - que vieram a definir novas direções na discussão dos efeitos quânticos inerentes às teorias de campos definidas em dimensionalidades mais altas.

A década de 1990 avança ainda com grande interesse na influência, via efeitos quânticos, das dimensões extras e sua compactificação no mecanismo da quebra eletrofraca e em processos que realizavam testes de precisão da Teoria Eletrofraca. Importante relembrar que, no período 1989-2000, o acelerador LEP do CERN encontrava-se no pleno de suas operações, e a era dos testes de precisão do setor eletrofraco constituía-se em uma grande questão deste período. Daí, o grande estímulo de se tentar detectar indiretamente as dimensões extras através de seus efeitos quânticos.

Já na reta final da década de 1990, Arkani-Hamed, Dimopoulos e Dvali quebram a quase monotonia da área: publicam, em 1998, uma série de artigos em que relacionam o problema da hierarquia de gauge do Modelo-Padrão a uma nova escala - sub-milimétrica para as dimensões extras, tirando-as da escala de Planck (ARKANI-HAMED; DIMOPOULOS; DVALI, 1998, 1999, 2002). Na proposta destes autores - que passou a ser conhecida como Large Extra Dimensions as novas dimensões compactas e sub-milimétricas são responsáveis pelo enfraquecimento da força gravitacional relativamente à força fraca na escala sub-nuclear. Este foi um passo muito inovador para a área, que, até então, sempre preconizou a escala Planckeana para as dimensões extras. Logo em seguida, em 1999, Randall e Sundrum introduzem um novo cenários para as dimensões extras (RANDALL; SUNDRUM, 1999), ao qual passou-se a ser referido como warped dimensi- 


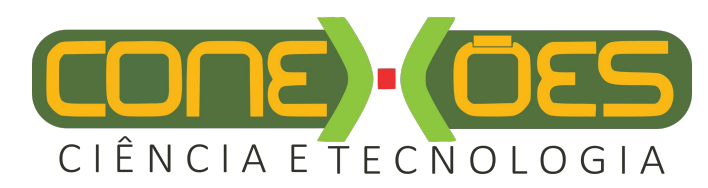

A RELATIVIDADE GERAL E A BUSCA DE NOVAS DIMENSÕES

ons: o que estes autores propõem é que os testes ligados à força gravitacional não impedem que sejam consideradas não-compactas (no modelo 5-dimensional a quinta dimensão pode ser infinita) as dimensões extra ao nosso Universo quadridimensional; este ponto de vista é realmente um corte no pensamento da área. E toda a ideia está baseada na existência de uma geometria não-fatorada, na qual a métrica do espaço-tempo quadridimensional passa a depender das dimensões extras. Quebra-se, então, neste cenário, a premissa de que a geometria do espaço-tempo completo seja fatorável, podendo, agora, termos a presença das coordenadas espaciais extras na própria métrica do espaço-tempo quadridimensional.

Inicia-se, com Randall e Sundrum, a fase das chamadas warped geometries e cenários de brane-world, com muitas aplicações ao estudo de efeitos quânticos nos processos envolvendo as partículas do ModeloPadrão e às consequências dos brane-worlds na formulação de modelos cosmológicos. Na concepção das warped geometries, nosso Universo é um espaço de anti-de Sitter 5-dimensional, no qual todas as partículas do Modelo-Padrão estão localizadas em uma brana quadridimensional, enquanto os grávitons propagamse e têm a sua dinâmica no interior do mundo 5dimensional. A partir daí o modelo de RandallSundrum se consolidou e vem sendo regularmente discutido na literatura da área. Em conexão com os modelos de Randall-Sundrum, é oportuno chamar atenção para um outro tipo de compactificação: a compactificação com fluxos em teorias de cordas (GRAÑA,2006), onde além da métrica, o espaço de dimensões extras também exibe um fluxo constante, associado a um campo magnético. E este tipo de compactificação dá suporte aos modelos do tipoRandall-Sundrum.

Na linha dos efeitos quânticos em teorias de campos em mais altas dimensões, poderíamos citar, em seguida à ampla aceitação das warped geometries, os trabalhos de Álvarez e Faedo, que também demarcam uma fronteira no tópico das correções quânticas (ÁLVAREZ; FAEDO, 2006, 2007). Nestes trabalhos, os autores atacam frontalmente a questão de comparar os procedimentos de quantização e cômputo de correções a 1-loop no espaço-tempo completo e no espaço-tempo reduzido com a inclusão das torres de campos massivos. Colocam em evidência as condições sob as quais as duas metodologias podem ou não ser equivalentes. Nos casos em que a inequivalência quântica fica comprovada, faz-se importante rever uma série de trabalhos da literatura onde se tratou a questão como se a equivalência fosse natural, como ocorre a nível clássico. Estes traba- lhos merecem a devida atenção se o foco é a discussão sobre como computar correções quânticas para modelos com mais dimensões.

Mais recentemente, podemos citar a linha de trabalhos iniciada por Bauman e Dienes e, em especial, no artigo de 2012 (BAUMAN; DIENES, 2012), onde aplicam um método de regularização que propuseram em 2008 (BAUMAN; DIENES, 2008a, 2008b) para o cálculo de efeitos radiativos em teorias com dimensões compactas. No trabalho de 2012, computam e demonstram explicitamente como as correções a 1-loop, calculadas no setor de modos massivos de Kaluza-Klein, alteram as relações entre massas e constantes de acoplamento. O trabalho tem consequências para o estudo de processos em Física além do Modelo-Padrão.

Há que se ressaltar a introdução de uma dimensão extra em uma questão bastante atual da Física de Matéria Condensada: o comportamento de uma nova categoria de materiais, denominados supercondutores topológicos, ainda em fase de síntese em laboratório. Para descrever a dinâmica dos elétrons e fótons neste material em nossas 3 dimensões espaciais, o interessante trabalho de Qi-Witten-Zhang (2013) introduz uma quarta dimensão espacial e formula um modelo eletrodinâmico em $(1+4)$ dimensões e adicionam à ação Maxwelliana o termo topológico de Chern-Simons, que, no mundo (1+3)-dimensional, induz naturalmente o termo tipoaxiônico, fundamental para a compreensão de propriedades dos supercondutores topológicos. Consideramos interessante citar este trabalho, pois abre um novo horizonte de dimensões extras em sistemas de Matéria Condensada.

Além destas questões mais recentes, gostaríamos de citar nossa proposta original de realizar a conexão entre um estudo semi-clássico (cálculo de potenciais interpartícula) realizado em dimensões superiores e a sua redução ao mundo quadridimensional. $\mathrm{O}$ esquema em questão, denominado de restrição dimensional (OSPEDAL, HELAYËL-NETO 2018) consiste no cálculo de potenciais inter-partícula em $(\mathrm{D}+\mathrm{N})$, com $\mathrm{D}$ sendo a dimensão do espaço-tempo de base e $\mathrm{N}$ o número de dimensões espaciais suplementares. $\mathrm{O}$ procedimento está baseado na premissa de que a interação entre as partículas no mundo quadridimensional não transfere momento nas $\mathrm{N}$ dimensões extras, ou seja, a interação fica restrita ao espaço-tempo D-dimensional. Neste cenário, as interações monopolo-monopolo permanecem inalteradas e aparecem correções no setor de spin. Isso se deve ao fato de que o conceito de spin é sensível a dimensionalidade. Por exemplo, no caso da interação eletromagnética, descrita pela lagrageana de 


\section{(CORE) (DES \\ CI ÊNCIA E TECNOLOGIA}

A RELATIVIDADE GERAL E A BUSCA DE NOVAS DIMENSÕES

Maxwell (com D=4 e N=1), obtêm-se com a restrição o conhecido potencial de Coulomb e novas correções no setor de quadrupolo.

$\mathrm{Na}$ linha de introdução de uma quinta dimensão não-compacta tipo-tempo com o propósito de se obter resultados físicos nas quatro dimensões espaçotemporais que experimentamos, citamos uma série de trabalhos publicados por Horwitz e colaboradores (LAND; HORWITZ, 2013), à qual se refere a literatura como Off-shell Quantum Electrodynamics. É uma abordagem interessante, tendo como consequência pequenos efeitos de correção para processos eletrodinâmicos usualmente calculados no âmbito da QED.

Até aqui, havíamos comentado dimensões 10, $11 \mathrm{e}$ 12 como as mais altas aparecendo na literatura. Entretanto, antes de concluirmos a nossa exposição, chamamos atenção para a linha de trabalho intitulada Double Field Theory, introduzida por Barton Zwiebach. Na qual o número de dimensões espaçotemporais é duplicado, de tal forma a tornar manifesta a chamada simetria-T. Assim, a teoria de cordas em 10 dimensões é formulada como uma teoria de campos em 20 dimensões com a dualidade-T explícita. Para uma introdução a esta interessante geometria, citamos estas notas de aulas de um curso do próprio autor (ZWIEBACH,2011) e o artigo de revisão (ALDAZABAL,MARQUES,NÚÑEZ,2013).

Finalizando, esperamos ter oferecido ao leitor interessado nas teorias à la Kaluza-Klein e na física que se pode extrair de dimensões espaço-temporais suplementares um panorama com seu desenvolvimento na linha do tempo e elencando as referências fundamentais associadas aos passos que foram dados para se estabelecer a ampla literatura na área das dimensões extras, assunto que permanece no fronte de interesses da Física de fronteira na área das Interações Fundamentais e Altas Energias.

Como fechamento, colocamos em destaque o fato de que a introdução de dimensões extras na Física de Interações Fundamentais vem possibilitando um notável progresso em dois programas de grande relevância na contemporaneidade: a quantização da gravidade e a unificação dos campos de força da Natureza, para os quais os mecanismos de redução e compactificação dimensional são de máxima importância. Esta foi a grande motivação que nos levou a contribuir com este texto neste volume de comemoração do Centenário do grande evento de Sobral.

\section{AGRADECIMENTOS}

L.P.R. Ospedal agradece o Conselho Nacional de Desenvolvimento Científico e Tecnológico
(CNPq/MCTIC) pelo suporte financeiro (bolsa PCI$\mathrm{DB})$.

\section{REFERÊNCIAS}

ABBOT, B. P. et al. (LIGO Scientific Collaboration and Virgo Collaboration, 2016), Phys. Rev. Lett. 116, 061102 (2016).

ALDAZABAL, G., MARQUES, D. e NÚÑEZ, C. Class. Quantum Grav. 30, 163001 (2013).

ÁLVAREZ, E.; FAEDO, A. F. JHEP 05 (2006) 046; Phys. Rev. D 74, 124029 (2006).

J. Phys. A: Math. Theor. 40, 6641 (2007).

APPELQUIST, T.; CHODOS, A. Phys. Rev. Lett. 50, 114 (1983).

Phys. Rev. D 28, 772 (1983).

APPELQUIST, T.; CHODOS, A. FREUND, P. G. O. "Modern Kaluza-Klein Theories", Addison-Wesley Pub. Co. 1987.

APPELQUIST, T.; CHODOS, A.; MYERS, E. Phys. Lett. 127B, 51 (1983).

ARE'FEVA, I. Ya.; VOLOVICH, I.V. Phys. Lett. 164B (1985) 287.

ARKANI-HAMED, N.; DIMOPOULOS, S.; DVALI, G. Phys. Lett. B429, 263 (1998).

\section{Phys. Rev. D 59, 086004 (1999).}

Physics Today, 35 (2002).

BAUMAN, S. DIENES, K. S. Phys. Rev. D 77, 125005 (2008).

Phys. Rev. D 77, 125006 (2008).

Phys. Rev. D 85, 125011 (2012).

BROCKILL, P. Cordas BPS Não-Abelianas e Uma Análise Simplética da Redução de Legendre, Tese de Doutorado, CBPF (2006).

CANDELAS, P.; HOROWITZ, G.; STROMINGER, A.; WITTEN, E. Nucl. Phys. B 258, 46 (1985).

CANDELAS, P.; WEINBERG, S. Nucl. Phys. B 237, 397 (1984).

CHODOS, A. Comments Nucl. Part. Phys. 13, 171 (1984a); 


\section{CORE) (DES \\ CI ÊNCIA E TECNOLOGIA}

A RELATIVIDADE GERAL E A BUSCA DE NOVAS DIMENSÕES

\begin{abstract}
"Quantum Aspects of Kaluza-Klein Theories", Yale University Preprint YTP/84-05 (1984b).
\end{abstract}

CREMMER, E.; SCHERK; J. Nucl. Phys. B 103, 393 (1976)

$$
\text { Nucl. Phys. B } 108 \text { (1976) } 409 .
$$

DELBOURGO, R.; WEBER, R. O. Il Nuovo Cim. 92A (1986) 347.

DIRAC, P.A.M. Proc. Roy. Soc. A 133, 60 (1931).

DIRAC, P. A. M. Journal of Mathematical Physics 4, 901 (1963).

DUFF, M. "The World in Eleven Dimensions - A Tribute to Oskar Klein", arXiv:hep-th/0111237v1.

DOLAN, L.; DUFF, M. J. Phys. Rev. Lett. 52 (1984) 14.

DUFF, M. J. NILSSON, B. E. W.; POPE, C. N. Phys. Rep. 130 (1986) 1.

EINSTEIN, A. The field equations of gravitation, Preussische Akademie der Wissenschaften, Sitzungsberichte 1915 (Part 2) 844.

EINSTEIN, A. Cosmological considerations in the General Theory of Relativity, Preussische Akademie der Wissenschaften, Sitzungsberichte 1917 (Part 1) 142.

EINSTEIN, A.; BERGMANN, P. Ann. Math. 39, 685 (1938).

FOCK, V. Zeits. für Phys. 39, 226 (1926).

FOSTER, J. G.; MÜLlER, B. Physics With Two Time Dimensions, arXiv:1001.2485 [hep-th].

FREUND; P. G. O.; RUBIN; M. A. Phys. Lett. 97B (1980) 233.

HUGGINS, S. R.; TOMS, D. J. Nucl. Phys. B 263 (1986) 433.

KALUZA, T. Sitzungsber. Preuss. Akad. Wiss. Berlin, Math. Phys. K1 (1921) 966.

KETOV, S. V.; NISHINO, H.; GATES Jr, S. J. Nucl. Phys. B393, 149 (1993).

GRAÑA, M. Phys. Rept. 423, 91 (2006).

GREEN, M. B. e SCHWARZ, J. H. Phys. Lett. 149 B, 117 (1984).
—. Phys. Lett. 151 B, 21 (1985).

KIBBLE, T. W. B. J. Math. Phys. 2, 212 (1961).

KLEIN, O. Talk "On the theory of charged fields" presented in the Meeting "New Theories in Physics", organized by the International Union of Physics, Warsaw (1938).

Zeits. für Phys. 37, 895 (1926).

LAND, M; HORWITZ, L. P. Journal of Physics: Conference Series 437, 012011 (2013).

MALDACENA, J. M. Adv. Theor. Math. Phys. 2, 231 (1998).

MANDEL, H. Zeits. für Phys. 39, 136 (1926).

NAHM, W. Nucl. Phys. B 135, 149 (1978).

NORDSTRÖM, G. Phys. Zeitschr. 15, 504 (1914).

ORZALESI; C. "Multidimensional Unified Theories", University of Parma Preprint IFPR/TH/63 (1980);

Fortsch. Phys. 29, 413 (1981).

OSPEDAL, L.P.R.; HELAYËL-NETO, J.A., Phys.Rev. D 97, 056014 (2018).

Efeitos de Spin, Velocidade e Dimensionalidade em Potenciais Inter-partículas Associados a Modelos de Gauge. Tese de Doutorado, CBPF (2017).

QI, X.-L.; WITTEN, E.; ZHANG, S.C. Phys. Rev. B 87 134519, (2013).

RANDALL, L.; SUNDRUM, R. Phys. Rev. Lett. 83 (1999) 3370; Phys. Rev. Lett. 83, 4690 (1999).

RANDJBAR-DAEMI, S; PERCACCI, R. Phys. Lett. 117B, 41 (1982).

SALAM, A.; STRATHDEE, J., Ann. Phys. (NY) 141, 316 (1982).

SAKHAROV, A. D. Sov. Phys. JETP 60, 214 (1984).

—. Zh. Eksp. Teor. Fiz. 87, 375 (1984).

. Sov. Phys. Usp. 34, 409 (1991).

. Usp. Fiz. Nauk 161, 94 (1991).

SCHERK, J.; SCHWARZ, J. H. Phys. Lett. 57B, 464 (1975).

Phys. Lett. 82B, 60 (1979)

Nucl. Phys. B 153, 61 (1979).

Phys. Lett. 84B, 83 (1979). 


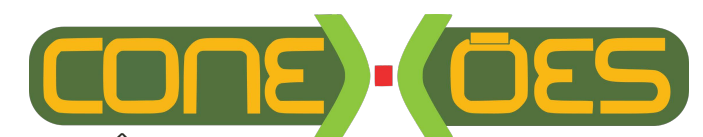

CI ÊNCIAETECNOLOGIA

A RELATIVIDADE GERAL E A BUSCA DE NOVAS DIMENSÕES

SHAW, R. "The Problem of Particle Types and Other Contributions to the Theory of Elementary Particles", Ph.D. Thesis (Part II, Chapter 3), Cambridge (1954).

SOHNIUS, M. F.; STELLE, K. S.; WEST, P. C. Nucl. Phys. B 173 (1980) 127; Phys. Lett. 92B (1980) 123.

UTIYAMA, R. Phys. Rev. 101 (1956) 1597.

VAFA, C. Nucl. Phys. B 469, 403 (1996).

WEIGAND, T. “TASI Lectures on F-Theory”, arXiv:1806.01854v1 [hep-th].

WITTEN, E. Nucl. Phys. B 186 (1981) 412.

- "Some Comments on String Dynamics", arXiv:hep-th/9507121v1.

Adv. Theor. Math. Phys. 2 (1998) 253.

YANG, C. N.; MILLS, R. L. Phys. Rev. 94, 191 (1954).

YUKAWA, H. Proc. Phys. Math. Soc.Japan 17, 48 (1935).

ZWIEBACH, B. "Double Field Theory, T-dualities and Courant Brackets", arXiv:1109.1782v1 [hep-th]; 